If a still smaller length has to be fashioned, every endeavour should be made to leave some part of the head of the humerus. The scar should not be on the anterior or posterior aspect of the stump because pressure is exerted on these surfaces in using the arm. A terminal scar from equal anterior and posterior flaps is ideal, with again little tissue in the flaps and a conical stump. Adhesions of the musculature to the end of the humerus must be avoided. This latter consideration is the chief objection to guillotine operations, which are otherwise satisfactory.

The length of the lower-arm amputation is measured from the tip of the olecranon to the amputated end of the ulna, and should be about 7 in. with the elbow flexed. The radius should be at least half an inch shorter than the ulna to obviate crossunion, and a conical stump be produced for the comfort of the patient and to permit him to use the prosthesis. The scar should be terminal in a line across the ends of the bones, and the flaps should contain no superfluous tissue. The nerves must be cut across cleanly to avoid the "phantom hand."

If an amputation has to be carried out with a shorter length than that suggested above, it will not prevent the amputee from using an artificial arm if at least three and a half inches is allowed to remain, but in such a case more muscle tissue must be trimmed away and a real bony stump left. If as a result of an accident, disunited fracture, or other cause a surgeon is confronted with a disarticulation of the elbow- or wrist-joint, an ideal site amputation is preferable, because these disarticulations do break down repeatedly. Furthermore, artificial arms must have a rotary mechanism at the wrist, and an above-elbow prosthesis will have the elbow-joint together with a rotary arm mechanism. These points are often forgotten when disarticulation amputations are performed.

In all arm amputations the patient should be encouraged to use the proximal joints and truncated muscles as early as possible. Crêpe bandaging with 3 -in. bandages is used when the stitches are removed. An arm amputee usually has a more despondent outlook than a leg case. It is for this reason that such cases should be fitted with prostheses as quickly as possible, otherwise there is a tendency to rely too much on the remaining hand. An arm can in the average case be fitted and used within a month of the operation.

Amputations of parts of the hands which have suffered trauma are indeed difficult. If any two working digits, or the thumb or part of it with a digit, can be saved it should be done to form the basis of a working hand. It is, however, surprising what appliances can be fitted to mutilated hands which may have only the minimum of a digit.

\section{Amputations in Children}

Children may have a limb amputated as a result of trauma, bone disease, congenital deformation, or aerial bombardment. These cases should all be treated early, for a child will quickly learn to use a leg or an arm with great ability. A plaster pylon or peg-leg should not be advised for the use of a child.

\section{Summary}

Records compiled by the Ministry of Pensions during the past 25 years definitely show that periodic examinations of amputated stumps are desirable.

The most suitable and trouble-free lengths of stumps, the position of the resultant scar from correctly fashioned skin flaps, and the treatment of the severed nerve are indicated.

In the past, stumps have received but little supervised attention with regard to their preparation for the fitting of a proc:?:esis.

A new method of crêpe bandaging is now used, the results of which prove its efficacy in reducing the oedema in a much shorter time than by previous methods. Pylons are not necessary, generally speaking. A course of exercises assists in preparing the stump musculature to control the artificial limb.

Arm amputations, not being weight-bearing, necessitate a different position for the scar. The stumps require bandaging and exercises to encourage the greatest range of joint mobility. The artificial arm should be fitted as soon as possible.

Children should not be fitted with non-articulated peg-legs. They should have a prosthesis at an early age, whether they be arm or leg cases.

\section{RENAL IMPAIRMENT DUE TO CRUSHING LIMBS IN ANAESTHETIZED DOGS}

BY

M. GRACE EGGLETON, D.Sc., M.R.C.S.

\author{
K. C. RICHARDSON, M.Sc. \\ H. O. SCHILD, M.D., Ph.D. \\ AND
}

\section{F. R. WINTON, M.A., M.D., D.Sc.}

Impairment of renal function in dogs under nembutal anaesthesia ensues immediately after a period of 4 to 5 hours, during which both hind limbs have been tightly bound with rubber tubing from ankle to hip and the thigh muscles compressed in a vice and hammered for a few minutes. Removal of the bandage is followed by a large fall in arterial pressure, and by either anuria or severe oliguria. In the latter event the urine is deeply pigmented, presumably with myohaemoglobin, which is freely excreted by the normal kidney, and the creatinine clearance is very low, which might be attributed in part to the low arterial pressure. Restoration of the arterial pressure with intravenous gum-saline or ox serum usually initiates or increases the urine flow; but even if the flow is raised to its initial value with diuretics the creatinine clearance, and therefore the concentrating power of the kidney, recovers to only about one-quarter of its original value.

The low arterial pressure following release of the limbs is not responsible per se for the damage to the kidney, since maintenance of even a lower blood pressure for an hour by means of a histamine infusion does not involve reduction in creatinine clearance during a subsequent period at normal arterial pressure. The reduction in urine flow after release of the limbs does not in itself account for the renal damage, since prevention of this reduction by diuretics does not materially affect the degree of the ultimate damage.

The damaged kidneys respond to large doses of diuretics with large increases in urine flow but only small increases in creatinine clearance, which may reach about one-half the normal value. In these respects we did not detect a significant difference in the efficacy of intravenous administration of hypertonic sodium chloride, isotonic sodium chloride, sodium bicarbonate, or sodium sulphate. The depth of colour of the urine from damaged kidneys was not consistently related to the severity of the damage as indicated by fall in creatinine clearance. The nature of the pigment was not examined in this series of experiments. Dr. Rimington has, however, identified the pigment in urine secreted under similar conditions in a later series as mainly myohaemoglobin.

Post mortem there was extensive injury to and oedema of thigh muscles, but no fracture of bones. The kidneys showed a brown cortex and a distinctively bluish-pink medulla, often with a narrow light-coloured zone between the two. The kidneys were notably flaccid, in contrast with the tense kidneys normally found after experimental poisoning.

Microscopically, the kidneys were characterized by the presence of material within the lumina of the tubules which might have been capable of blocking some of the tubules. The material was particularly concentrated in the terminal portion of the proximal convoluted tubules. The distal and collecting tubules were usually free and distended. There was, however, no consistent relation between the extent of the deposit and the impairment of renal function; and, indeed, extensive deposit occurred in some kidneys which were functionally undamaged. In the only dog in which the anuria could not be antagonized even by huge doses of diuretics there were mitochondrial changes in the proximal tubule cells, indicating the action of a poisoning agent.

\section{Discussion}

In these observations on 21 dogs we used the reduction in creatinine clearance as the chief indicator of renal damage because, like the inulin clearance, it is probably a measure of the glomerular filtration rate in the dog unless there is leakage of 
creatinine from the concentrated urine in the tubules back into the blood. The renal damage described above is probably not due mainly to reduction in glomerular filtration rate, because oliguria from this cause is associated with very high creatinine and low chloride concentration in the urine, whereas in these experiments the creatinine concentrations are low and the chlorides may be increased. Microscopically the damaged organ appears fairly vascular.

Blockage of the tubules, suggested as a possible factor by the appearance of the deposit in them, should produce a tense kidney, in contrast with the striking flaccidity observed in these damaged kidneys. The deposit does not appear compact enough, nor does it occur in a sufficiently high proportion of tubules, to lead one to expect that it could reduce the creatinine output to one-quarter. A more likely hypothesis would involve release of a poison from the damaged limbs that affects the tubule cells by increasing their permeability to creatinine, urea, and other substances which are concentrated by the kidney. These substances would diffuse from the concentrated solution in the distal parts of the tubules across the tubule walls to the blood in the venules. Hastening the flow of liquid down the tubules with diuretics should reduce the loss by leakage of creatinine and so increase the creatinine clearance, an expectation which accords with observation. The main objection to regarding this as a self-sufficient hypothesis is that poisons such as cyanide or mercury salts produce an increased urine flow, due to inhibition of water reabsorption, before they produce the anuria due to increased permeability. Correspondingly, organs poisoned in this way are usually tense. This is, however, not an insuperable objection to the view that the poison here in question affects the permeability mainly.

Such a poison might reach the tubule cells either from the blood stream or, if diffusible, from the lumen of the tubule. In the latter event the poison itself might well be a substance which is concentrated in the tubules, and act the more potently in virtue of its concentration. This possibility has, however, been excluded in the following way. Partial obstruction of one kidney by an inerease of ureter pressure resulted in a fourfold concentration of urine (as evidenced by creatinine- $U / P$ ratio) compared with the unobstructed side. If the toxic agent released from the limbs had been similarly more concentrated on the obstructed side the severity of the damage should have been greater on that side. In fact, the obstructed side, after removal of the obstruction, was, if-anything, a little less severely damaged than the unobstructed side.

So far as available evidence goes, the injury to the kidneys in our series of dogs appears to be of the same kind as that in man after comparable prolonged crush injury to the limbs. The essential nature of the renal damage has not yet been determined, but the evidence suggests that the main factor may be concerned with increase in permeability of the renal tubules due to a toxic agent released from the damaged limbs, while there may be an additional factor involving reduction in the rate of glomerular filtration probably due to lowering of glomerular capillary pressure.

\section{VARIATIONS IN THE RESPIRATORY RHYTHM OF PROGNOSTIC SIGNIFICANCE IN MALIGNANT HYPERTENSION BY}

\section{H. O. GUNEWARDENE, M.B., B.S., D.M.R.E. Radiologist, General Hospital, Colombo}

Recently I drew attention to variations in the respiratory rhythm in a case of malignant hypertension in its terminal stages (Gunewardene, 1938). A similar type of respiration has been noticed in cases of meningitis (Hutchison and Hunter, 1929). The characteristic feature is that the respirations are grouped; in each group the initial excursion in the tracing is the largest, the subsequent ones getting smaller, until the movements cease for a moment. This means that the depth of the respiration gradually declines as in the waning phase of Cheyne-Stokes respiration. In that case, however, there was no period of apnoea.
This type of respiration is seen in the terminal stage of malignant hypertension when pronounced albuminuria, persistent hypertension, and extensive eye changes (haemorrhages, exudates, cotton-wool patches, oedema of the disks, etc.) are present. The patient is generally quiet and lies flat on the back without a suggestion of the breathlessness of the cardiac type which previously had been well marked. A little restlessness, not continuous, is often observed; he opens his mouth frequently and breathes as though he is yawning half-heartedly. Occasionally he is irrational. Sometimes there is maniacal excitement; at.other times a little drowsiness suggestive of a uraemic state. Uraemic twitching or convulsions were not observed.

These variations in the respiratory rhythm in association with malignant hypertension have not been described before, so far as I can gather from the literature available in Ceylon. Their cause has yet to be explained. Are they due to cerebral oedema, or to the effects of toxins on the respiratory centre? Is the alteration in the chemistry of the blood responsible for them? Eyster has shown that Cheyne-Stokes breathing can be produced in dogs by a rise and fall of the blood pressure in the presence of raised intracranial pressure.

In the following case, grouping of respiratory movements similar to those in the case referred to above was noticed, but each group was followed by a period of apnoea. The tracing is unlike Biot's type, but resembles that of Cheyne-Stokes respiration with the waxing phase absent. The rhythm is irregular and the depth of the respirations varies.

\section{Case Report}

The patient, a male sedentary clerical' worker aged 42 , was admitted on March 7, 1939, complaining of occasional occipital headache, giddiness on and off, and blurring of vision. He was lying quiet, but a few days previously he had been very dyspnoeic. Examination revealed the following characteristics. Heart: heaving apex beat-in fifth space, midclavicular line : second aortic ++ ; no murmurs. The lungs were clear. Blood pressure: systolic 300 , diastolic 180 . The brachial and radial

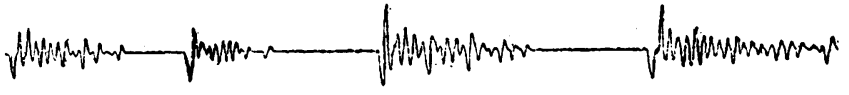

arteries did not feel much thickened, nor did palpation suggest such high pressure. Pulse 78 , regular. Urine: scanty; S.G. 1010 ; albumin $0.7 \%$; no sugar. Fundi showed oedema of the disks ; flame-shaped haemorrhages, exudates, thickened arteries, very congested veins. Blood urea, $224 \mathrm{mg}$. per $100 \mathrm{c.cm}$. ; urea clearance, mean $7.26 \%$ normal.

Treatment consisted of venesection ; administration of sodium nitrite, theominal, etc.

During his stay in hospital the blood pressure varied between $210 / 140$ and $260 / 170$ (not eliminating effects of drugs). He became somewhat irrational, yawned half-heartedly, and later became a little drowsy and quiet. Just before his death (under ayurvedic treatment) on April 7, 1939, he complained that he "couldn't breathe." The accompanying illustration shows the respiratory tracing (thoracic) of this case.

According to Harrison (1939), the carbon dioxide tension in malignant hypertension is abnormally low and patients with periodic breathing have arterial blood which is under-ventilated as regards oxygen and over-ventilated as regards carbon dioxide. It has also been shown that the administration of carbon dioxide abolishes periodic breathing. The fairly sudden transition of a dyspnoeic, sometimes orthopnoeic, patient into a condition of tranquillity would suggest that changes in the chemical constituents of the blood gases and the tissue fluids play a significant part in the production of periodic breathing and the cessation of dyspnoea.

\section{Summary}

A form of variation in the respiratory rhythm associated with malignant hypertension is described.

There are changes in the periodicity as well as the depth of the respiratory movements.

These changes are of grave prognostic significance even when the patient does not look seriously ill, the duration of life in the cases described being not more than 6 weeks after their onset. 